\title{
TRACKING DIRECTION-OF-ARRIVAL FOR WIRELESS COMMUNICATION WITH MULTIPLE ANTENNAS
}

\author{
Dmitriy Shutin and Gernot Kubin \\ Graz University of Technology, Infeldgasse 16c, A-8010, Austria \\ dshutin@inw.tugraz.at, kubin@inw.tugraz.at
}

\begin{abstract}
The goal of this contribution is to bind together results available for radar systems for Direction-of-Arrival (DOA) tracking and wireless communications with multiple antennas. We show that for accurate tracking of DOA's the MUSIC-like methods based on inversion of the channel snapshot covariance matrix are suboptimal. In our approach we apply an alternative algorithm developed by $\mathrm{H}$. Gu(2002) that uses the instant channel snapshot to update the DOA estimates directly. We improve on this algorithm by introducing online data association capabilities based on linear extrapolation that leads to better tracking performance and lower computational complexity. The performance is evaluated using simulated channels as well as real wideband Multiple-Input-Multiple-Output (MIMO) measurements at $2 \mathrm{GHz}$. The obtained estimates confirm previous results obtained with alternative techniques but provide more accurate results at lower computational complexity.
\end{abstract}

Keywords: MIMO, DOA tracking algorithm, mobile communication.

\section{INTRODUCTION}

Wireless systems are subject to fading - time variations of the receiving conditions that are caused by multipath propagation and transceiver movements. Recently, the study of MIMO antenna systems in communication has shown their superior potential to fight fading by exploiting smart antennas and space diversity techniques [1].

One of the possible approaches to compensate fading is channel prediction based on past channel measurements[2]. Basically, this technique assumes the channel impulse response to be modeled by a certain time-varying model. For the time being, these techniques were developed for Single-Input-Single-Output (SISO) models $[3,2]$. In the SISO case, a channel tap $h_{k}$ could be locally (in time) expressed as a sum of complex sinusoids

$$
h_{k}(t)=\sum_{n=1}^{N} c_{k, n} \mathrm{e}^{j\left(w_{d, n}(t) \cdot t\right)}
$$

where $w_{d, n}(t)$ 's are the Doppler frequencies of $N$ different resolvable scatterers or reflectors, and $c_{k, n} \in \mathbb{C}$ are complex amplitudes. Since the wireless channel is completely characterized by the reflectors and scatterers distributed around the transceiver, the accurate parametrization of this geometry would result in a more accurate description of the channel and, therefore, more accurate predictions.
In the SISO case, the only information available about the nearby objects is their Doppler shifts and complex attenuation factors. Multiple antennas, however, also preserve directions of incoming wavefronts. This provides a richer description of the channel behavior and additional degrees of freedom in designing communication systems. In the following we restrict ourselves to the Single-Input-Multiple-Output (SIMO) case, thus considering only estimation of Directions-of-Arrival (DOA's). Most of DOA estimation algorithms rely on properties of the sensor covariance matrix [4]. We show that for accurate channel modeling in wireless communication, these methods are not only computationally expensive, but also might be suboptimal, since they require the stationarity of the moving objects. This is hardly the case for SIMO wireless channels. Recently $\mathrm{H}$. Gu [5] has proposed an algorithm to update the DOA estimates which does not require estimation of the sensor covariance matrix. However, this algorithm becomes unstable when several objects have the same or similar DOA's. Here we propose a method to resolve these instabilities by online prediction of such situations and locally substituting DOA estimates with linear extrapolations.

The remainder of the paper is organized as follows: in section 2, we will present an extension of the SISO description to the SIMO case; section 3 gives a short review of the DOA estimation and tracking algorithm, section 4 deals with some simulation results, and, finally, in section 5 we treat the application of these ideas to real MIMO channel measurements conducted by Forschungszentrum Telekommunikation Wien, Austria.

\section{SAMPLED WIRELESS SIMO CHANNEL}

In [2] it was shown that the time-varying channel tap $h_{k}(t)$ could be represented as

$$
h_{k}(t)=\sum_{n=1}^{N} \alpha_{k, n}(t) \mathrm{e}^{j \psi_{n}(t)}
$$

where $N$ is the number of resolvable multipath components within the channel excess delay bin $k$, and

$$
\begin{aligned}
\alpha_{k, n}(t)= & g\left(T k-\tau_{n}(t)\right) a_{n}(t) \mathrm{e}^{j\left(\zeta_{n}(t)-w_{c} \tau_{n}^{\text {const }}\right)}, \\
& \text { and } \psi_{n}(t)=-\frac{2 \pi}{\lambda}\left\|\boldsymbol{r}_{n}^{v a r}(t)\right\| .
\end{aligned}
$$

Here, $\alpha_{k, n}(t)$ accumulates all slowly time-varying components, such as the attenuation $a_{n}(t)$ due to the free space propagation,

\footnotetext{
${ }^{1}$ Authors wish to thank Forschungszentrum Telekommunikation Wien for providing MIMO channel measurements data.
} 
filtering effect at the transmitter, $g\left(T k-\tau_{m}(t)\right)$, with symbol period being $T$, the phase shift due to the scatterer/reflector structure, $\mathrm{e}^{j \zeta_{n}(t)}$, and the phase shift due to the time-invariant propagation delay $\tau_{n}(t)$ (base-station - reflector). The carrier frequency is $w_{c}$. On the other hand, the phase term $\psi_{n}(t)$ stands for the electrical distance of the time-varying path $\boldsymbol{r}_{n}^{v a r}(t)$ (reflector - mobile). This term is the source of the small-scale fading that occurs even as the mobile moves a fraction of the wavelength.

Now we consider the change in the electrical distance $\psi_{n}(t)$ for a single wave source as the antenna array moves with constant velocity $\boldsymbol{v}$ (i.e. SIMO case). The roles of the array and reflectors/scatterers in this setup could always be interchanged due to the reciprocity of the channel.

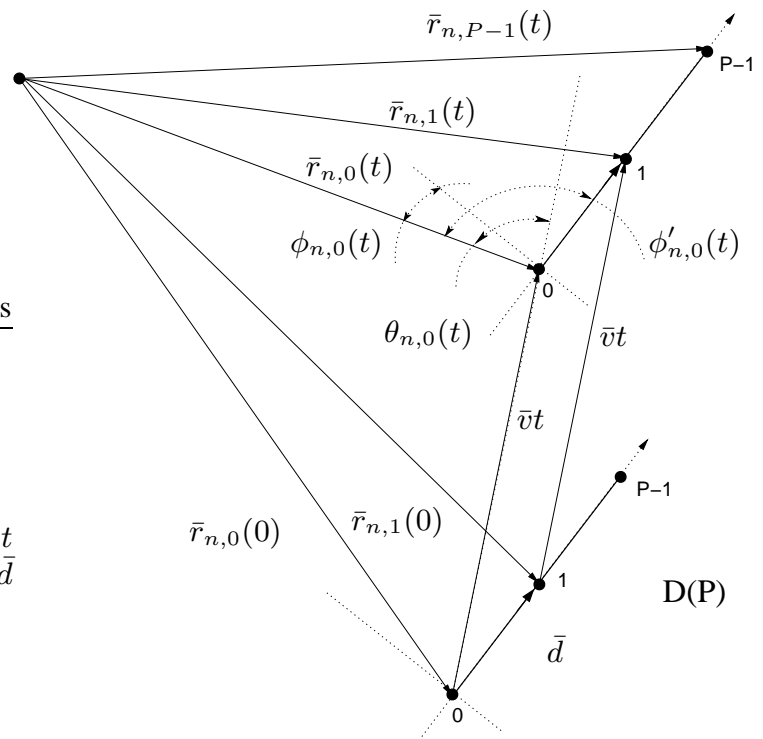

Fig. 1: Instantaneous phase, SIMO case.

For the sake of simplicity, we assume that the receiving antenna $D(P)$ is a simple linear array with $P$ sensors. Consider the situation depicted in Fig.1. In vector form, the $p$-th distance to the antenna $\boldsymbol{r}_{n, p}(t)$ is given as follows.

$$
\boldsymbol{r}_{n, p}(t)=\boldsymbol{r}_{n, p}(0)+\boldsymbol{v} t=\boldsymbol{r}_{n, 0}(0)+p \boldsymbol{d}+\boldsymbol{v} t
$$

For large distances, i.e., under the plane wave assumption, the length of $\boldsymbol{r}_{n, p}(t)$ is found to be:

$$
\begin{aligned}
\left\|\boldsymbol{r}_{n, p}(t)\right\| & =\left\|\boldsymbol{r}_{n, 0}\right\|-p\|\boldsymbol{d}\| \cdot \cos \left(\phi_{n, 0}^{\prime}(t)\right)- \\
& -\|\boldsymbol{v}\| t \cdot \cos \left(\theta_{n, 0}(t)\right)
\end{aligned}
$$

Combining (2),(4) and (5) we get the final expression for the channel tap in the SIMO case:

$$
\begin{gathered}
h_{k, p}(t)=\sum_{n=1}^{N} \tilde{\alpha}_{k, n}(t) \mathrm{e}^{j \cdot w_{d, n}(t) t} \cdot \mathrm{e}^{j \cdot \frac{2 \pi}{\lambda} p \cdot\|d\| \cdot \sin \left(\phi_{n}(t)\right)} \\
\text { where } w_{d, n}(t)=\frac{2 \pi}{\lambda}\|\boldsymbol{v}\| \cdot \cos \left(\theta_{n, 0}(t)\right) \\
\text { and } \tilde{\alpha}_{k, n}(t)=\alpha_{k, n}(t) \cdot \mathrm{e}^{-j \frac{2 \pi}{\lambda}\left\|\boldsymbol{r}_{n, 0}\right\|}
\end{gathered}
$$

$w_{d, n}(t)$ is the Doppler shift induced by the $n$th wave source, and $\phi_{n, 0}(t)$ is the corresponding DOA. Under the plane wave assumption, all rays become parallel and induce the same DOA and the same Doppler shifts for all the sensors in the array. It is also important to note from (6) that for different antennas the channel tap variations differ only in the phase shift induced by the DOA's of the wave impinging on the array. This brings us to the issue of tracking the DOA's from different scatterers/reflectors.

\section{DIRECTION-OF-ARRIVAL ESTIMATION}

DOA estimation for multiple moving targets has been addressed in radar array processing since the 1960 s. For simplicity, the problem is formulated for a linear array of $P$ sensors. Let $\boldsymbol{h}$ be the complex-valued $P$-dimensional data vector, obtained from stacking the outputs of $P$ sensors at the time $t$. Then

$$
\boldsymbol{h}=\sum_{n=1}^{N} \boldsymbol{s}\left(\phi_{n}\right) a_{n}+\boldsymbol{\eta}(n)=\mathbf{S}(\boldsymbol{\phi}) \boldsymbol{a}+\boldsymbol{\eta}(n)
$$

where $\mathbf{S}(\boldsymbol{\phi})=\left[\boldsymbol{s}\left(\phi_{1}\right), \boldsymbol{s}\left(\phi_{1}\right), \ldots, \boldsymbol{s}\left(\phi_{N}\right)\right]$ is a $P \times N$ matrix, $N<P$, composed from steering vectors $\boldsymbol{s}\left(\phi_{n}\right)=$ $\left[1, \mathrm{e}^{j d_{1} \xi_{n}}, \ldots, \mathrm{e}^{j d_{P-1} \xi_{n}}\right], \xi_{n}=(2 \pi / \lambda) \sin \left(\phi_{n}\right) ; \lambda$ is the wavelength, and $d_{p}$ is the distance between the first and the $p$ th sensor. In Eq.(7), $\boldsymbol{\phi}=\left[\phi_{1}, \ldots, \phi_{M}\right]$ are the unknown DOA's of $N$ different wave sources, $\boldsymbol{a}=\left[a_{1}, \ldots, a_{M}\right]$ is a complex-valued return vector, and $\boldsymbol{\eta}(t)$ is a complex-valued Gaussian white noise. It is known that all the steering vectors $\boldsymbol{s}_{m}(\phi)$ are orthogonal to the $(P \times N)$ dimensional "noise" subspace of the covariance matrix $\mathbf{R}=\left[\boldsymbol{h} \boldsymbol{h}^{H}\right]$, spanned by all eigenvectors associated with the smallest singular values[6]. MUSIC-based estimates of DOA's are formed by picking the $N$ values of $\phi_{n}$ that minimize the projection of $\boldsymbol{s}(\boldsymbol{\phi})$ onto this noise space[4]. The model (6) could be easily rewritten in terms of (7) by the following substitutions:

$$
\begin{aligned}
\boldsymbol{s}\left(\phi_{n}\right) & =\left[1, \mathrm{e}^{j \xi_{n}}, \ldots, \mathrm{e}^{j(P-1) \cdot d \cdot \xi_{n}}\right]^{T}, \\
\xi_{n} & =(2 \pi / \lambda) \sin \left(\phi_{n}(t)\right) \\
\boldsymbol{a} & =\left[\alpha_{k, 1} \mathrm{e}^{j w_{d, 1}(t) \cdot t}, \ldots, \alpha_{k, N} \mathrm{e}^{j w_{d, n}(t) \cdot t}\right]^{T}
\end{aligned}
$$

It is easy to note that in this case the amplitude $\boldsymbol{a}$ is timedependent. Covariance-based methods require stationarity of moving objects at least within the analysis window needed to estimate the covariance matrix R. According to Eq.(8) this is, however, not the case in SIMO wireless channel. The Doppler shifts vary at least as fast as the corresponding DOA's, and thus, the amplitude $\boldsymbol{a}$ in (8) would vary even faster. MUSIC-based estimates that do not yield optimal results in general[7], might produce even wrong estimates in the case of wireless SIMO or MIMO channels. Therefore, in tracking the wireless channel we have to rely only on the current snapshot to estimate and update the DOA's. H. Gu [5] has developed a radar tracking algorithm for multiple moving targets, that does not require estimation and inversion of the covariance matrix $\mathbf{R}$, which is a computationally heavy task. The algorithm computes Instantaneous Maximum Likelihood Estimates (IMLE) of the DOA's given an instantaneous data snapshot. Moreover, the algorithm achieves the Cramer-Rao bound in estimating $\phi$ given $\boldsymbol{h}$ at moment $t$. The significant drawback of the algorithm is that if DOA trajectories for different moving objects intersect, then the algorithm looses stability, since the steering matrix $\mathbf{S}$ in (7) becomes ill-conditioned. We propose a method to mitigate this by introducing an "inertia" concept for the tracking system. Such property 
will allow the system to move beyond the intersection point with the tracking algorithms switched off. We have implemented this mechanism by means of linear extrapolation. While estimating the DOA's, we observe the gradient over a certain memory span, and use this information to solve for the approximate trajectory intersection point. In the vicinity of this point we switch off the estimator algorithm and continue the DOA trajectories along their gradient, exchanging the estimator outputs with the linear extrapolator output. The pseudo-code that implements this approach on the base of Gu's tracking algorithm is shown in Table.1.

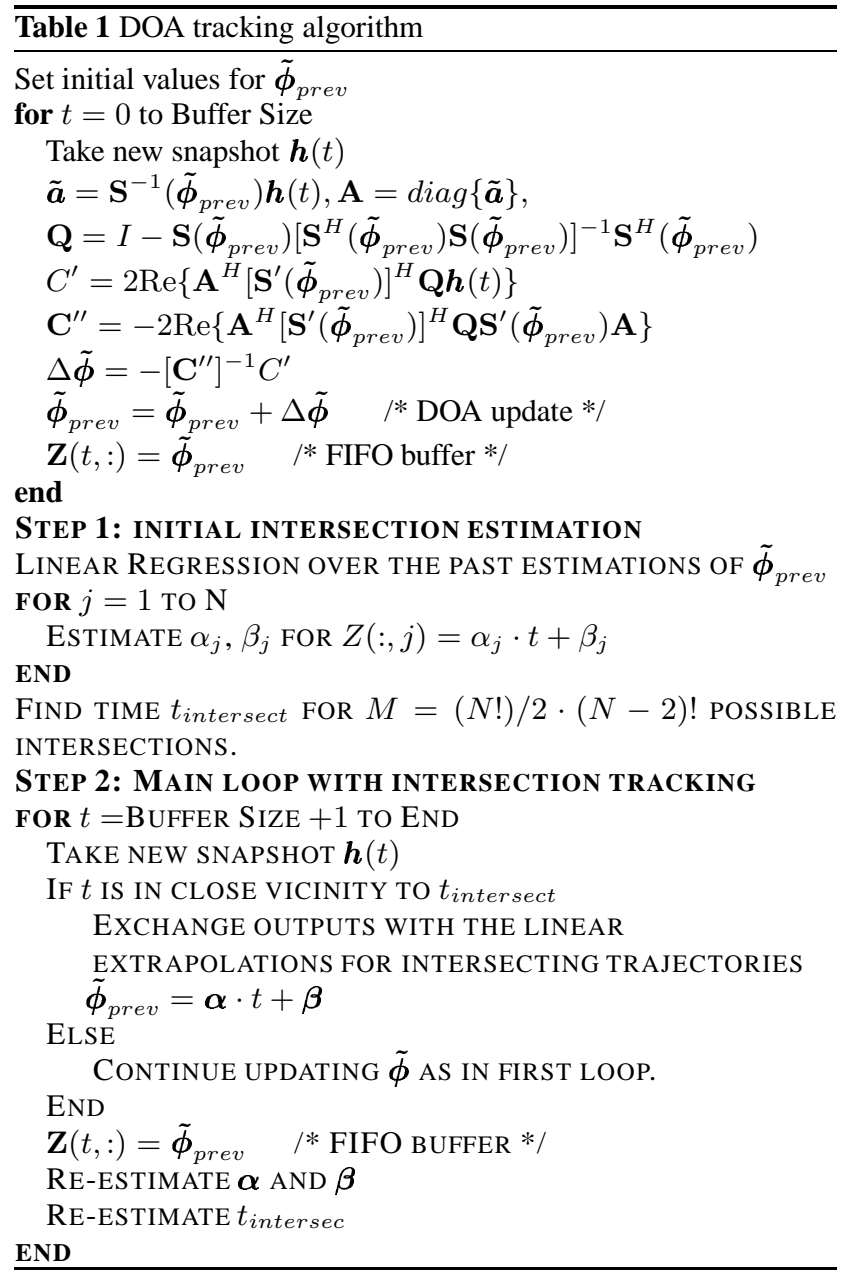

The matrix $\mathbf{S}$, with $\mathbf{S}^{\prime}$ being its derivative and $\mathbf{S}^{-1}$ being its pseudoinverse, is defined exactly as in (7). The "close vicinity" of $t$ to $t_{\text {intersec }}$ can be empirically inferred from values of the receiver noise and variance of the estimates.

\section{SIMULATIONS}

A simple simulation model can be easily derived from the equations (6) and (3). The following simple setup was used: the receiving linear antenna array with $P=10$ sensors is positioned at the origin of the coordinate system. The sensor spacing is $d=0.15 \mathrm{~m}$ at the half of the wavelength(Fig.2). Reflectors are distributed in the upper semiplane.

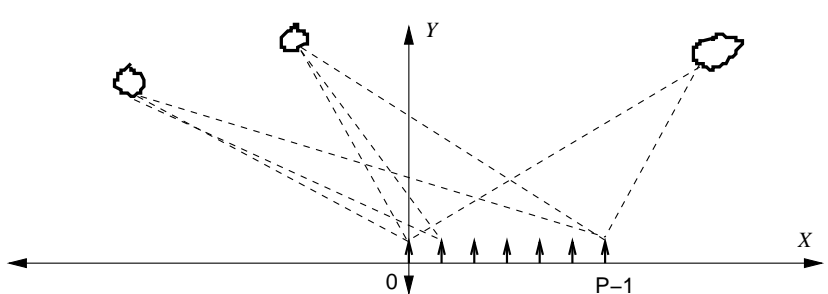

Fig. 2: Simulation setup

\begin{tabular}{lll}
\hline \hline Exper. & $\mathrm{A}$ & $\mathrm{B}$ \\
\hline \hline Distances & $R_{1}=500 \mathrm{~m}$ & $R_{1}=800 \mathrm{~m}$ \\
& $R_{2}=500 \mathrm{~m}$ & $R_{2}=500 \mathrm{~m}$ \\
\hline Initial angle & $\phi_{1}(0)=45^{\circ}$ & $\phi_{1}(0)=45^{\circ}$ \\
& $\phi_{2}(0)=20^{\circ}$ & $\phi_{2}(0)=-30^{\circ}$ \\
\hline Velocities & $20 \mathrm{~m} / \mathrm{s}$ at $10^{\circ}$ & $30 \mathrm{~m} / \mathrm{s}$ at $10^{\circ}$ \\
& $40 \mathrm{~m} / \mathrm{s}$ at $10^{\circ}$ & $50 \mathrm{~m} / \mathrm{s}$ at $170^{\circ}$ \\
\hline SNR & $11 \mathrm{~dB}$ & $11 \mathrm{~dB}$ \\
\hline \hline
\end{tabular}

Table 2: Simulation Parameters

We generate a channel tap that includes two multipath components and an additive complex Gaussian white noise. The simulation scenarios are shortly summarized in the Table.2. Angles are measured with respect to the $Y$-axes (i.e. antenna's broadside) for reflectors, and with respect to the $X$-axes (i.e. antenna's endfire) for velocities. In these experiments the SNR is defined as:

$$
S N R=10 \log _{10} \frac{\sum_{l}\left|h_{l}\right|^{2}}{\sum_{l}\left|n_{l}\right|^{2}}
$$

where $h_{l}$ is the generated complex channel tap for one of the array sensors in the $l$ th channel snapshot and $n_{l}$ is the corresponding complex noise value. Experiment A, Fig.3, shows the estimated DOA trajectories for two reflectors, that are $500 \mathrm{~m}$ away from the antenna.

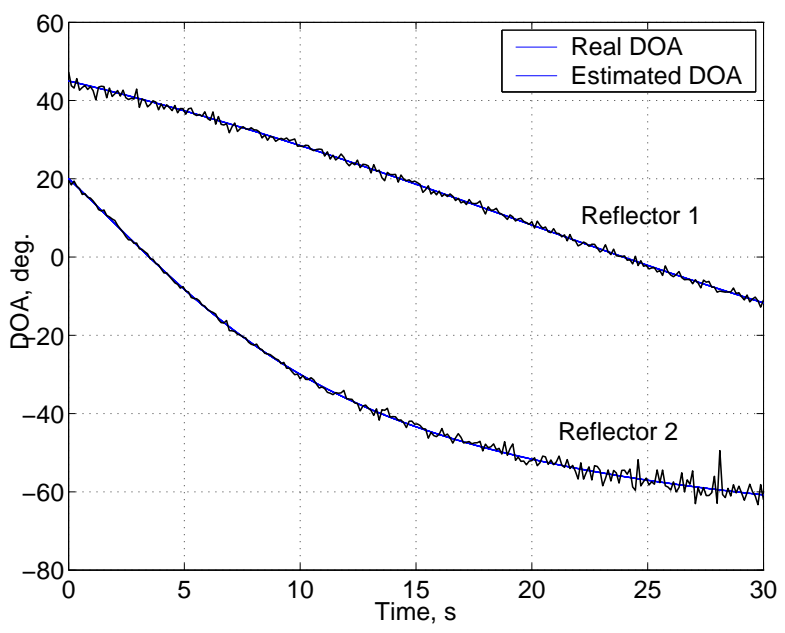

Fig. 3: Experiment A 


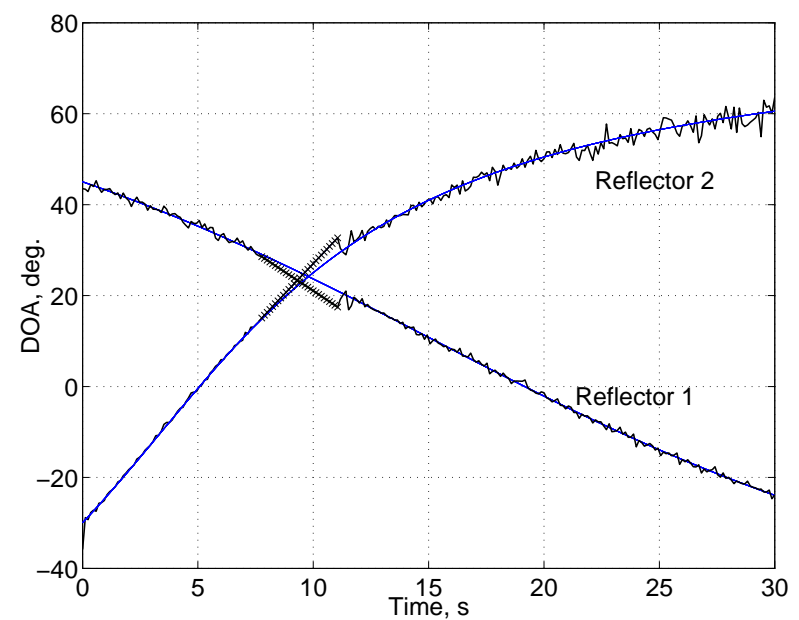

Fig. 4: Experiment B

For such a distance it is reasonable to assume that the angles of incidence, as well as Doppler shifts, are the same for all the sensors in the array. As we can see, the algorithms perform quite well even for considerably small SNR values.

\begin{tabular}{lll}
\hline \hline Distances & Initial angle & Velocities \\
\hline$R_{1}=14 \mathrm{~m}$ & $\phi_{1}(0)=60^{\circ}$ & $1.5 \mathrm{~m} / \mathrm{s}$ at $10^{\circ}$ \\
$R_{2}=7 \mathrm{~m}$ & $\phi_{2}(0)=20^{\circ}$ & $2.1 \mathrm{~m} / \mathrm{s}$ at $10^{\circ}$ \\
\hline \hline
\end{tabular}

Table 3: Close scatterers

The simulation depicted in Fig.4 shows a more complicated scenario when two DOA trajectories intersect. In the vicinity of the intersection point we switch off the tracking algorithm and continue the trajectory along the gradient, exchanging the outputs with the linear regression predictions (Fig.4). Although, such approach is not optimal for tracking DOA's in the close vicinity of the intersection point, it provides reasonable predictions of the initial angles beyond the intersection point to ensure fast re-convergence.

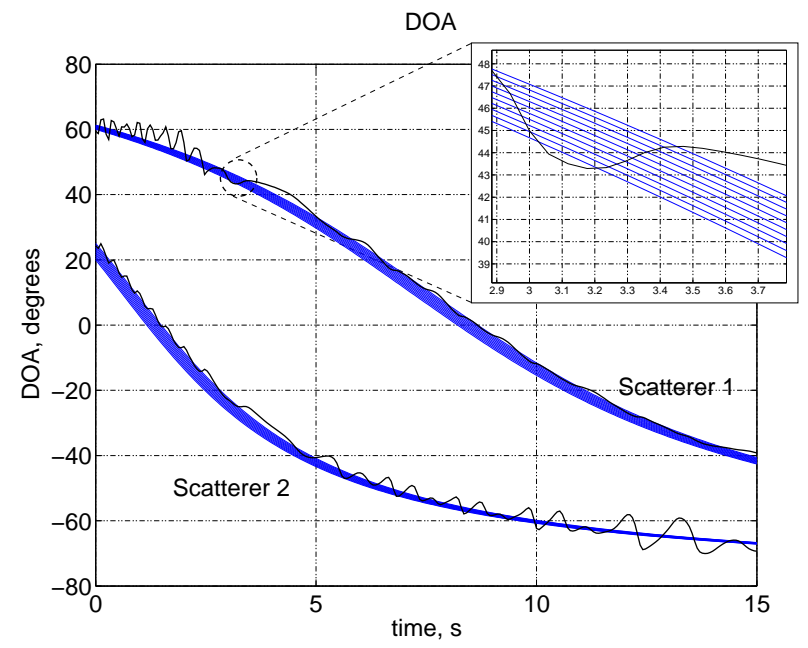

Fig. 5: Two close scatterers
The next experiment (Fig.5) shows the simulation of two close scatterers. The details are summarized in Table.3. In this experiment the SNR was deliberatly set to infinity to demonstrate the following particularity: in this setup every antenna sees a different angle, resulting in a thick belt on the plot. Generally speaking, in this case the model (6) is a very crude approximation to the real channel. It is possible to see that the estimated trajectories oscillate around the true DOA's quite significantly even for small angles. These oscillations result from uncertainty in estimating the steering vectors since they are no longer sampled complex exponentials but rather chirps which emerge from "seeing" different Doppler shifts and DOA's on different sensors in the array. Under the same initial conditions the oscillating pattern remains even in case of the lower SNR, which makes us belive it is the effect of proximity to the receiving antenna rather than numerical effects.

We conclude, that an algorithm for DOA tracking in the proximity of the antenna array should, in general, include preprocesing of the antenna snapshots by a certain spatial filtering to amend these effects.

\section{RESULTS FOR MIMO MEASUREMENTS}

The last three figures show the DOA tracking for MIMO channel sounder measurements which were performed by Forschungszentrum Telekommunikation Wien, FTW, Vienna, Austria, under supervision of Helmut Hofstetter[8]. The measurements were done with the MIMO capable wideband vector channel sounder RUSKATM, manufactured by MEDAV [9]. The sounder was specifically adapted to operate at the center frequency of $2 \mathrm{GHz}$. The transmitted signal is generated in the frequency domain to ensure a predefined spectrum over $120 \mathrm{MHz}$ bandwidth, and an approximately constant envelope over time. Two simultaneously multiplexed antenna arrays have been used at the transmitter and receiver. The transmitter was a uniform circular array, with 15 sensors spaced at $\approx 6.45 \mathrm{~cm}$ and placed on the roof of a racing car. The car velocity was $\approx 160 \mathrm{~km} / \mathrm{h}$. The receiver was a fixed uniform linear array, with 8 antenna elements spaced at a distance of $\lambda / 2 \approx 7.5 \mathrm{~cm}$. For our purposes we select only a SIMO subset, by taking only one transmitting antenna from the array.

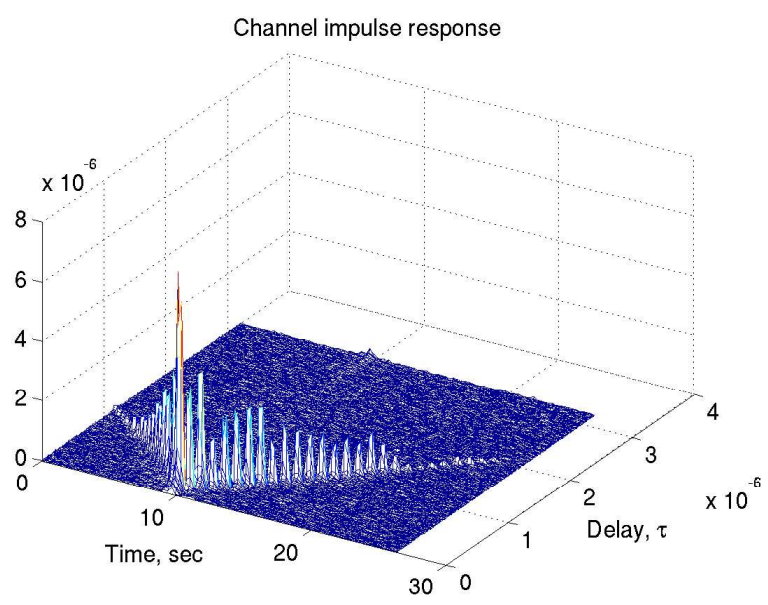

Fig. 6: SIMO channel impulse response $h(t, \tau)$ 


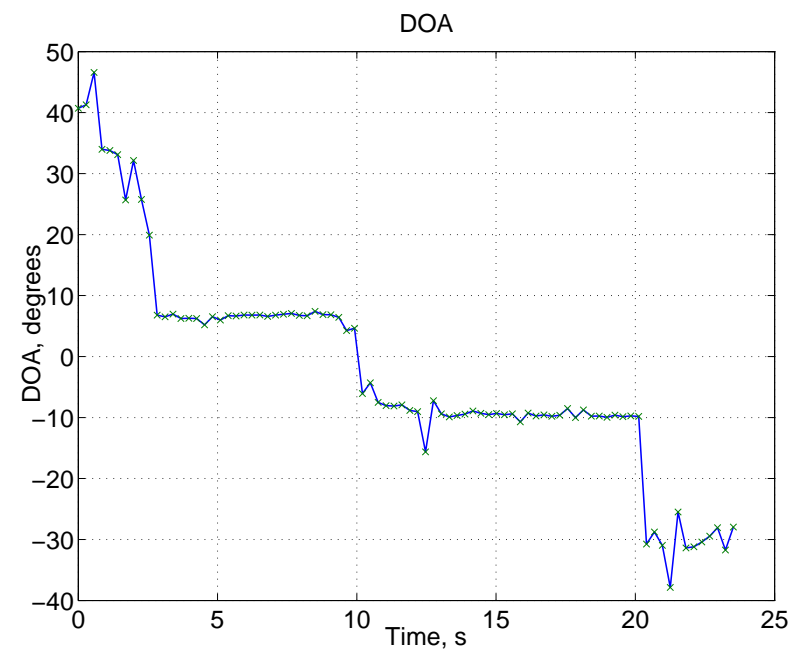

Fig. 7: DOA trajectory of a single strongest component

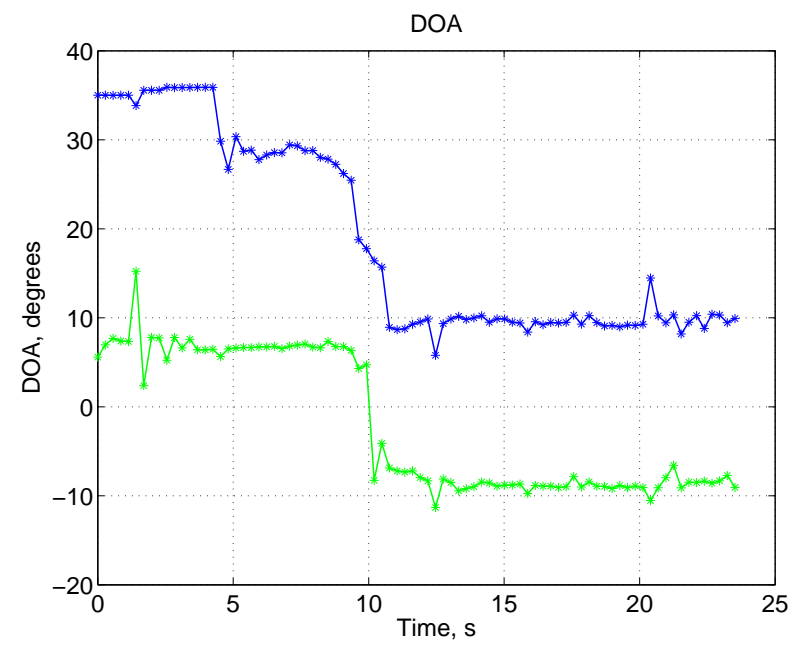

Fig. 8: DOA trajectories of the two resolved strongest multipath components

Figure 6 shows the measured channel impulse response as the car passes the receiver. It is easy to observe the car approaching the receiver and passing by. In the measured channel, there is a strong LOS component, however, there are also several strong reflections[8]. At such high speeds, the impulse response changes very fast (Fig.6). The same reflector or scatterer appears in different delay bins as the car moves. Here, we only track the strongest components that correspond to the LOS-component and the second strongest reflection. In Fig.7, the estimated DOA trajectory is shown. These estimates were obtained with the prior of only a single contribution to the tap. Fig. 8 shows the same measurement, but for two components contributing to the tap. Our estimates confirm results obtained by [8], however our approach is computationally much more efficient. In general, the complexity of MUSIC is of the order $P^{3}$, where $P$ is the size of the array. Gu's algorithm has complexity of only $N^{3}+N^{2} P$ (see [5]), where $N$ is the number of the strongest multipath components. Thus, for the described measurements this algorithm is $512 / 40=12.8$ times more efficient.

\section{CONCLUSION}

We have shown that the theoretical basis for application of MUSIC-like methods to estimating DOA's might be inadequate in case of wireless communication. The fact that Gu's algorithm achives Cramer-Rao bound makes us belive that our approach might be more efficient, however, the quantitative comparison of both algorithms under practical accuracy specifications must be further investigated. We have also extended Gu's tracking algorithm by online gradient-based predictions of DOA trajectory intersections. Simulations show that our algorithm could be successfully applied to low SNR cases to resolve intersecting DOA trajectories. The performance of the proposed approach to DOA tracking was also tested on real wireless MIMO channel measurements. The obtained results agree with previous results obtained with alternative MUSIC-based algorithms, however, the algorithm used in our experiments has much lower computational complexity.

\section{REFERENCES}

[1] G.J. Foschini and M.J. Gans, "On limits of wireless communications in a fading environment when using multiple antennas," Wireless Personal Communications, vol. 6, pp. 311-335, 1998.

[2] T. Ekman, Prediction of Mobile Radio Channels, Modeling and Design, Ph.D. thesis, Uppsala University, Nov. 2002.

[3] T. Ekman and G. Kubin, "Nonlinear prediction of mobile radio channels: Measurements and MARS model designs," IEEE Proc. on Acoustics, Speech, and Signal Processing, vol. 5, pp. 2667-2670, 1999.

[4] Harry L. Van Trees, Optimum Array Processing, Wiley, 2002.

[5] H. Gu, "Radar angle-tracking system for multiple moving targets," IEE Proc. on Radar, Sonar and Navig., vol. 149:1, pp. 23-28, Feb. 2002.

[6] R.O. Schmidt, A signal subspace approach to multiple emitter location and spectral estimation, Ph.D. thesis, Standford University, 1981, PhD dissertation.

[7] P. Stoica and A. Nehorai, "MUSIC, maximul likelihood, and Cramer-Rao bound: Further results and comparisons," IEEE Trans. Acoust., Speech, and Sig. Proc., vol. 38, pp. 21402150, 1990.

[8] E. Bonek et al., "Double-directional superresolution radio channel measurements," Proc. Conf. on Comm., Control, and Computing, vol. 3, Oct. 2001.

[9] R. Thoma et al., "Identification of time-variant directional mobile radio channels," IEEE Trans. on Instrumen. and Meas., vol. 49:2, pp. 357-364, Apr. 2000. 